\title{
Merging droplets in double nanocontact spin torque oscillators
}

\author{
Dun Xiao, ${ }^{1}$ Yaowen Liu, ${ }^{1, *}$ Y. Zhou,,${ }^{2,3, \dagger}$ S. M. Mohseni,${ }^{4,5,6}$ S. Chung,,${ }^{6,7}$ and J. Åkerman ${ }^{6,7, \dagger}$ \\ ${ }^{1}$ Shanghai Key Laboratory of Special Artificial Microstructure Materials and Technology, School of Physical Science and Engineering, \\ Tongji University, Shanghai 200092, China \\ ${ }^{2}$ School of Electronics Science and Engineering, Nanjing University, Nanjing 210093, China \\ ${ }^{3}$ Department of Physics, The University of Hong Kong, Hong Kong, China \\ ${ }^{4}$ Department of Physics, Shahid Beheshti University, Tehran 19839, Iran \\ ${ }^{5}$ NanOsc AB, Electrum 205, 16440 Kista, Sweden \\ ${ }^{6}$ Materials Physics, School of Information and Communication Technology, KTH Royal Institute of Technology, \\ Electrum 229, 16440 Kista, Sweden \\ ${ }^{7}$ Department of Physics, University of Gothenburg, 41296 Gothenburg, Sweden
}

(Received 11 December 2015; published 25 March 2016)

\begin{abstract}
We demonstrate how magnetic droplet soliton pairs, nucleated by two separated nanocontact (NC) spin torque oscillators, can merge into a single droplet soliton. A detailed description of the magnetization dynamics of this merger process is obtained by micromagnetic simulations: A droplet pair with a steady-state in-phase spin precession is generated through the spin-transfer torque effect underneath two separate NCs, followed by a gradual expansion of the droplets' volume and the out-phase of magnetization on the inner side of the two droplets, resulting in the droplets merging into a larger droplet. This merger occurs only when the NC separation is smaller than a critical value. A transient breathing mode is observed before the merged droplet stabilizes into a steady precession state. The precession frequency of the merged droplet is lower than that of the droplet pair, consistent with its larger size. Merged droplets can again break up into droplet pairs at high enough magnetic field with a strong hysteretic response.
\end{abstract}

DOI: 10.1103/PhysRevB.93.094431

Topological solitons in magnets [1,2] with spin textures have recently attracted considerable attention for spintronic applications [3-10]. Generally, the topological protection makes the lifetime of these nontrivial quasiparticles much longer than that of topologically trivial particles. Magnetic bubbles [11-13], domain walls [14,15], magnetic vortices [16-20], and skyrmions [6-9,21] are typically static topological solitons and have been well studied. Differing from these static structures, the dissipative magnetic droplet soliton (droplet hereafter) is nontopological, inherently dynamic, and results from the balance between anisotropy, exchange, spin-transfer torque, and magnetic damping [22,23].

Droplets have been experimentally reported in nanocontact spin torque oscillators (NC-STOs) having a free layer with strong perpendicular magnetic anisotropy (PMA) and an in-plane spin polarizer fixed layer [24-27]. In such NC-STOs [28-30] the energy dissipated by damping is compensated for by the energy input from the current-induced spin-transfer torque (STT) effect [31,32], so that the droplet is expected to be strongly localized in the $\mathrm{NC}$ region and to have its spins precessing in phase around the film normal with a very large precession angle. The droplet typically has a partially reversed magnetization directly underneath the $\mathrm{NC}$ and a zone of large amplitude precession in a region bounding the reversed magnetization, which can lead to an increase in the microwave output power of NC-STOs by a factor of 40 [25] compared to those of nondroplet counterparts.

\footnotetext{
*Author to whom correspondence should be addressed: yaowen@tongji.edu.cn

†yanzhouy@ hotmail.com

†johan.akerman@physics.gu.se
}

NC-STOs exhibiting droplets hence provide a promising route to achieving much larger signal-to-noise ratios, which comprise an essential prerequisite for their application [33]. Additionally, the controllability of droplets through current and field may provide a means for the transportation and storage of information with droplets as carriers in future spintronic systems [23,34], analogous to optical solitons in fiber optic communications.

In this paper, we report on the merging of two individually driven droplets into a single merged droplet by the combined action of an external magnetic field $H$ and a drive current $I$. We find that the merger depends both on the applied current and the magnetic field, as well as on the separation distance between the two magnetic droplets, with the existence of a critical distance $d_{c}$.

As shown in Fig. 1(a), we consider a NC-STO geometry based on a pseudo-spin-valve structure patterned into a rectangular shape with a long axis of $1024 \mathrm{~nm}$ and a short axis of $512 \mathrm{~nm}$. The fixed spin polarizer layer is assumed to be magnetized along the $+z$ direction and the 2-nm-thick free layer has perpendicular magnetic anisotropy (PMA). The free layer has two NCs, each with radius $r=50 \mathrm{~nm}$ and with a separation distance varied from 150 to $250 \mathrm{~nm}$. Positive current is defined as the flow of electrons from the free layer to the polarizer layer.

Micromagnetic modeling of the above design was performed using the open-source simulation software MUMAX3 [35], based on the Landau-Lifshitz-Gilbert equation including the STT term $[31,36]$ :

$$
\frac{d \mathbf{m}}{d t}=-\gamma \mathbf{m} \times \mathbf{H}_{\mathrm{eff}}+\alpha \mathbf{m} \times \frac{d \mathbf{m}}{d t}+a_{J} \mathbf{m} \times\left(\mathbf{m} \times \mathbf{m}_{p}\right),
$$


(a)
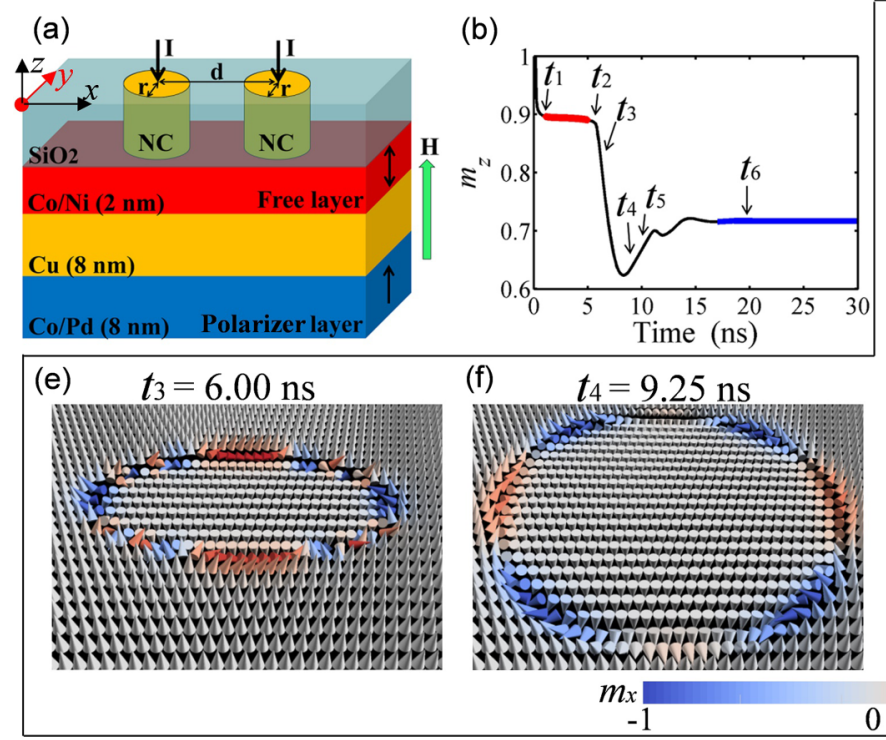
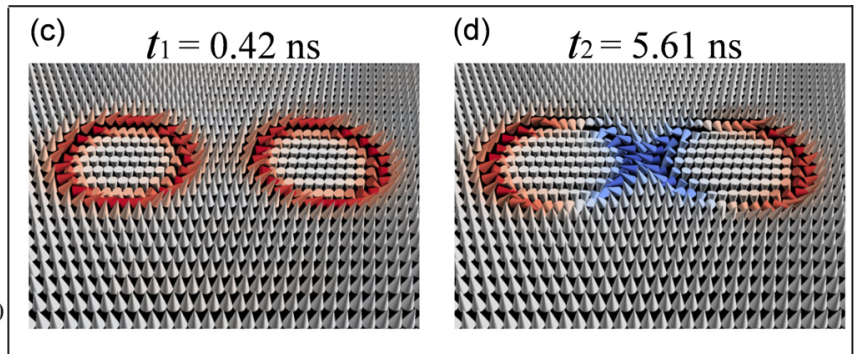

(g) $\quad t_{5}=10.30 \mathrm{~ns}$

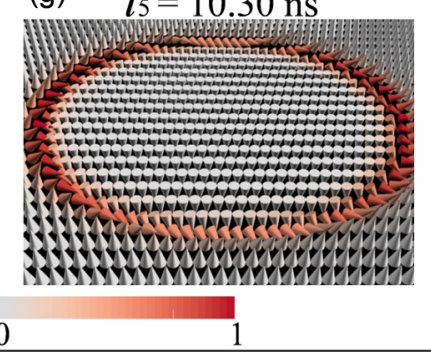

FIG. 1. Current- and magnetic-field-induced merging of a droplet pair. (a) Schematic of the NC-STO. A magnetic field is applied in the out-of-plane direction with a strength of $0.5 \mathrm{~T}$. The current through each $\mathrm{NC}$ is fixed at $I=-15 \mathrm{~mA}$. (b) Time evolution of the $z$ component of average magnetization. (c)-(h) Transient snapshots of magnetization for a small region around the NCs. The colored cones display the $x$ component of the magnetization.

where $\mathbf{m}=\mathbf{M} / M_{s}$ is the normalized magnetization of the free layer, $M_{s}$ is the free layer saturation magnetization, $\alpha$ is the Gilbert damping factor, and $\gamma$ is the gyromagnetic ratio. $\mathbf{H}_{\mathrm{eff}}=$ $-\left(1 / \mu_{0} M_{s}\right) \partial E / \partial \mathbf{M}$ is the effective magnetic field derived from the free energy density of the system. The energy includes the dipole-dipole interaction $E_{d}$, the exchange interaction parametrized by an exchange constant $A$, the PMA energy, and the Zeeman energy due to an applied magnetic field $\mathbf{H}$. The total energy is written as $E=E_{d}+A\left[\left(\partial_{x} \mathbf{m}\right)^{2}+\left(\partial_{y} \mathbf{m}\right)^{2}\right]-$ $\mathbf{M} \cdot \mathbf{H}-K_{u} m_{z}^{2}$. The last term of Eq. (1) describes the STT effect. The torque factor $a_{J}(\theta)=\hbar \gamma g(\theta) J /\left(2|e| M_{s} t\right)$, which depends on the current density $J$, the thickness of free layer $t$, and $g(\theta)$. Here $g(\theta)$ is a scalar function depending on the polarization of electrons $P$ and the angle $\theta$ between the magnetization vector of the free layer $\mathbf{m}$ and that of the polarizer layer $\mathbf{m}_{p}$. In this study, we assume that the current is injected only into the $\mathrm{NC}$ region and that the spin polarization is set to $P=1$. The following standard parameters are used for the free layer [37] $M_{s}=716 \mathrm{kA} / \mathrm{m}$ (saturation magnetization), $K_{u}=447 \mathrm{~kJ} / \mathrm{m}^{3}$ (magnetic anisotropy), $A=30 \mathrm{pJ} / \mathrm{m}$ (exchange stiffness), and $\alpha=0.01$ (Gilbert damping). The free layer is divided into $512 \times 256$ unit cells, corresponding to a cell size of $2 \mathrm{~nm}$, which is smaller than the exchange length $\left(\lambda_{\mathrm{ex}}=\sqrt{2 A / \mu_{0} M_{s}}=9.7 \mathrm{~nm}\right)$. All the simulations are performed at zero temperature.

A typical example of droplet pair creation and their transformation into a merged droplet is studied in Figs. 1(b)1(h), beginning from an initial uniform parallel configuration $\left(m_{z}=+1\right)$ with respect to the magnetization direction of the polarizer. The NC separation distance is $d=195 \mathrm{~nm}$, the applied magnetic field is $\mu_{0} H=0.5 \mathrm{~T}$ (i.e., $H=400 \mathrm{kA} / \mathrm{m}$ ), and current through each $\mathrm{NC}$ is $-15 \mathrm{~mA}$, resulting in a current density of $1.91 \times 10^{12} \mathrm{~A} / \mathrm{m}^{2}$. Figure 1 (b) shows the time evolution of the $z$ component of the average magnetization over the full free layer area. A series of transient snapshots taken from the magnetization evolution process is shown in Figs. 1(c)-1(h), which clearly displays the typical magnetization spatial distribution. The corresponding times are indicated in the temporal magnetization curve of Fig. 1(b). Under the action of the current-induced STT effect, the magnetization at the two $\mathrm{NC}$ regions first departs from the initial parallel state and rotates around the $z$ axis. Then, the magnetization at the center region of the NCs is switched to the negative $z$ direction, producing a magnetic droplet pair structure, as shown in Fig. 1(c) at $t_{1}$. Note that the two droplets have the same precession phase, i.e., they are mutually synchronized.

The two droplets continue to precess as individual droplets for about 5 ns. However, during this time, their radius gradually increases, as evidenced by the gradual reduction in $m_{z}$ in Fig. 1(b) (red line) until their boundaries interact and eventually touch. Owing to the relatively small separation distance, the spin precession underneath the NCs strongly affects the middle region between the droplet pairs through the exchange coupling and the dynamical dipolar interactions. However, unlike the spins underneath the NCs, the spins between the droplets are not directly driven by STT (no current flows in this zone). As a result, the precession phase is delayed to such a degree that the time-dependent magnetization at the inner side (blue color) of the two droplets is opposite in phase to that at the outer side [red color; see Fig. 1(d)]. This marks the onset of the two droplets merging to form a single droplet at the moment $t_{3}$; see Fig. 1(e). The topography of the $m_{z}$ component during the merging process is shown in the Supplemental Material (Fig. S1) [38]. As a result, the reversed magnetization area with $-m_{z}$ becomes much larger, leading to a sudden drop in the average $m_{z}$ curve, as shown in Fig. 1(b).

Interestingly, the precession phase of the spins at the perimeter of the newborn merged droplet exhibits blue-and-red 
(a)


FIG. 2. (a) Time trace of the magnetization averaged over the simulation area; the magnetization changes from a droplet pair precession orbital (red) to a merged droplet precession orbital (blue). (b)-(e) Magnetization $x$-component evolution for four typical procession states during the merger process.

stripes [out of phase; see Figs. 1(e) and 1(f)], indicating high wave vector perturbations of the perimeter spins. The calculated topological number for this type of merged droplet is, however, still zero. These strong, high wave vector perturbations are accompanied by strong breathing of the merged droplet. Over time, the blue-and-red stripes, the high wave vector perturbations, and the breathing are all damped out, leaving behind a stable droplet with a perimeter having all the spins precessing in phase [Fig. 1(g)].

In order to further investigate the internal mechanism of the merging process, the trace of the magnetization averaged over the full free layer for the same simulation is shown in Fig. 2(a), where the steady-state precession orbit of the droplet pair is indicated by the red curves and that of the merged droplet is indicated by the blue curves. Figures 2(b)-2(e) display the time evolution of the magnetization's $x$ component $m_{x}$ for four typical precession states, as described in Fig. 1, during the merging process. The perfect stable periodic precession of the droplet pair is given in Fig. 2(b), which corresponds to the state of Fig. 1(c). Figure 2(c) shows the process when the two droplets merge. The oscillation amplitude of the average $m_{x}$ decreases slightly when the droplets begin to interact. This is attributed to the fact that the magnetization oscillation in the middle region is opposite in phase to that of the outer side of the two NCs [Fig. 1(c)]. After that, the merged droplet's "breathing mode" state is clearly observable by the average $m_{x}$, as shown in Fig. 2(d). The moments at which the magnetization configurations of Figs. 1(f) and 1(g) are taken have been marked in Fig. 2(d). Finally, the merged droplet's breathing mode relaxes and the merged droplet goes into a steady-state periodic precession mode, which can be easily seen in Fig. 2(e).

Another interesting result is that the frequency of the merged droplet is a little lower than that of the initial droplet pair, as indicated in Fig. 3(a), showing that the frequency of the merged droplet is $16.0 \mathrm{GHz}$, while that of the original droplet (a)

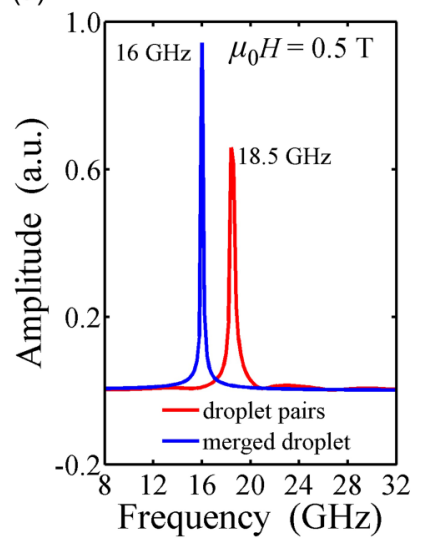

(b)

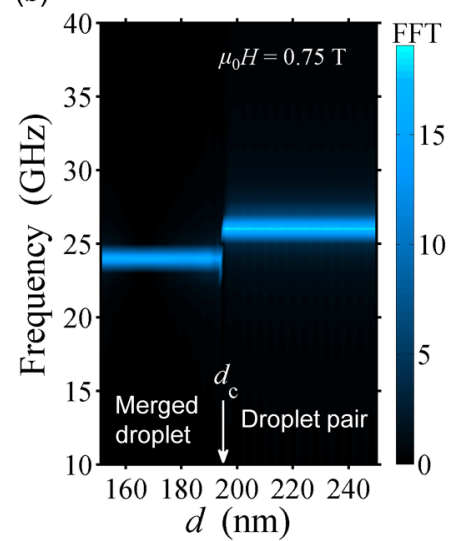

FIG. 3. (a) Frequency spectra of the droplet pair and the merged droplet calculated by FFT from the in-plane magnetization $m_{x}$. (b) Frequency diagram of final precession state for two NCs as a function of separation distance $d$.

pair is $18.5 \mathrm{GHz}$. Here the frequencies are calculated from the $m_{x}$ curves [Figs. 2(b) and 2(e)] using the fast Fourier transform (FFT) method. It should be pointed out that the amplitude of the frequency peaks in Fig. 3(a) calculated by the FFT has no obvious physical meaning. The somewhat larger value of the merged droplet is attributed to its relatively large perimeter, which gives rise to greater $x$ components of the magnetization. It is noticed that the values of the frequency agree well with the relation of solitons [39]:

$$
\omega=\omega_{0} \sqrt{\pi N_{0} / N} .
$$

Here, $\omega_{0}$ is the uniform ferromagnetic resonance frequency. $N=\frac{M_{s}}{2 \mu_{0}} \int\left(1-m_{z}\right) d^{3} x$ is the number of spin deviations, which can be calculated from the droplet profiles as shown in the Supplemental Material (Fig. S1). $N_{0}$ is the characteristic number of particles. From Fig. 3(a), the precession frequency of the droplet pair $\omega_{\text {pair }}$ is $18.5 \mathrm{GHz}$. For the droplet pair we have $N=0.7$ and for the merged droplet $N=0.9$. Thus, the theoretical prediction of the merged droplet frequency can be obtained from Eq. (2), $\omega_{\text {merged }}=0.886 \times \omega_{\text {pair }}=16.3 \mathrm{GHz}$, which is in good agreement with our simulation value of $16.0 \mathrm{GHz}$.

It is reasonable to assume that the merging of the droplet pair depends strongly on the separation distance between the two NCs. To understand how the separation influences the droplet merging process, we carried out a series of simulations at $\mu_{0} H=0.75 \mathrm{~T}$ by changing $d$ from 150 to $250 \mathrm{~nm}$. Figure 3(b) shows the separation distance dependence of the frequency spectra for the steady-state magnetization procession of the final states after $15 \mathrm{~ns}$ simulations. A critical value of the separation distance (here, $d_{c}=195 \mathrm{~nm}$ ) can be clearly observed, below which the droplet pair can merge into a merged droplet due to the strong exchange and dynamical dipolar interaction effects. The oscillation frequency of the merged droplet is $24 \mathrm{GHz}$, while the magnetic droplet pair oscillates at $26 \mathrm{GHz}$. 
(a)

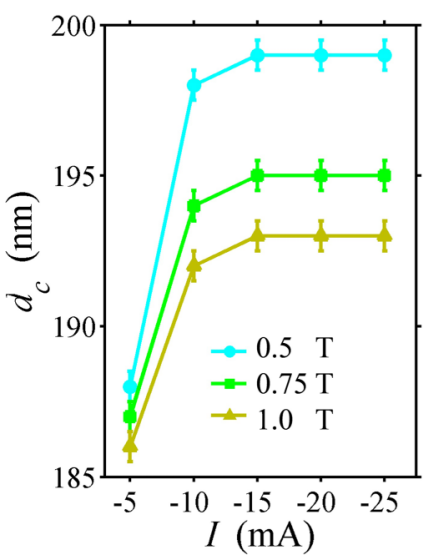

(b)

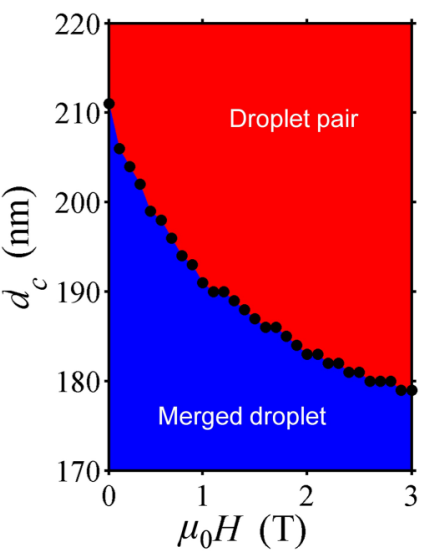

FIG. 4. (a) Critical separation distance $d_{c}$ as a function of current for merging a droplet pair into a merged droplet at three different magnetic fields. (b) Operational range as a function of magnetic field for a given current.

The critical separation $d_{c}$ plays an important role in the process of merging of the droplet pairs. We find that $d_{c}$ decreases with applied magnetic field and increases with current, and saturates at a sufficiently large current. Figure 4(a) shows the current dependence of $d_{c}$ for various values of the magnetic fields. Obviously, $d_{c}$ increases with current up to $-10 \mathrm{~mA}$, and afterwards maintains an almost constant value of 192-197 $\mathrm{nm}$ for the three different magnetic fields. This result indicates that the further local enhancement of the STT effect through increasing the current in the $\mathrm{NC}$ regions cannot increase the critical separation distance of the droplet pair merging. In contrast, decreasing the magnetic field could increase the threshold separation. A more detailed phase diagram of the dependence of $d_{c}$ on the magnetic field for a droplet pair (red region) and for a merged droplet soliton (blue region) is given in Fig. 4(b), simulated at a fixed current of $I=-15 \mathrm{~mA}$. The largest $d_{c}$ is $211 \mathrm{~nm}$ at zero magnetic field. The operational range for merging a droplet pair into a merged droplet is indicated in Fig. 4(b) by the blue-colored region.

The merger process can also be reversed by increasing the magnetic field to very high values. In other words, the merged droplet can be broken up and the original droplet pair recovered. This recovery process occurs at a comparative high applied field and the droplet pair locally stabilizes in the NC region. Figure 5 shows a typical recovery process of a droplet pair. The field sweep direction is indicated by the arrows, starting from zero field to $10 \mathrm{~T}$ and then back to zero. The blue (red) curve in Fig. 5 shows the response of $m_{z}$ during the $\mu_{0} H$ increasing (decreasing) process. The simulation starts from a merged droplet state. A fixed current of $-10 \mathrm{~mA}$ is applied in each NC. With the field increasing, the spins located in the middle "isolation" zone first reverse to the $+z$ direction, and form a ring-shaped configuration; see Fig. 5. However, this configuration is extremely unstable, and differently shaped configurations frequently change when the field increases from 3.5 to $7.5 \mathrm{~T}$. Accordingly, the $m_{z}$ curve fluctuates widely until a magnetic field larger than a

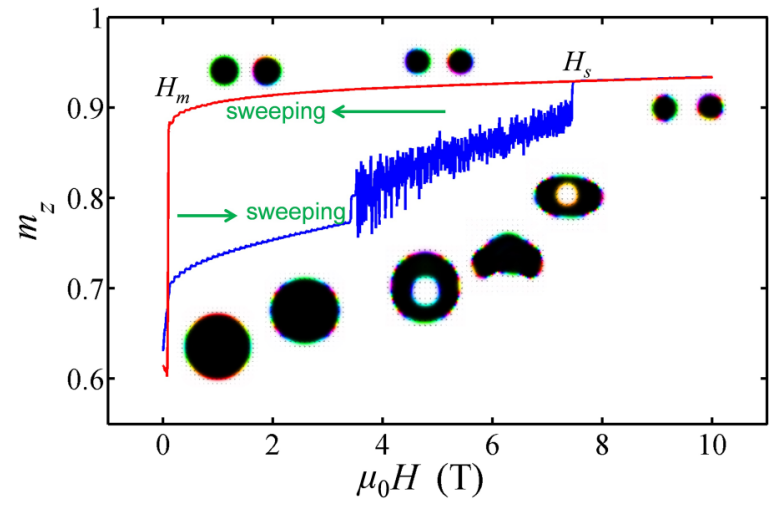

FIG. 5. Separation process of a merged droplet into a droplet pair. The blue and red curves show the $z$-component magnetization as a function of magnetic field sweeping loop. The applied current in each $\mathrm{NC}$ is fixed to be $-10 \mathrm{~mA}$. The typical magnetization configurations are shown by a series of snapshots taken during the recovery process.

critical magnetic field $\mu_{0} H_{s}(=7.5 \mathrm{~T})$ is reached, at which the merged droplet is completely separated into a pair of droplets. After that, this droplet pair remains until the magnetic field decreases down to $\mu_{0} H_{m}=0.2 \mathrm{~T}$, at which point the droplet pair merges again. We find that the critical field $\mu_{0} H_{s}$ required to separate the merged droplet strongly depends on the applied current, showing the decreased $\mu_{0} H_{s}$ for a smaller current (not shown).

Finally, we would like to point out that the initial synchronization state before the merger process is the key factor. Our further simulations indicate that if the two droplets are generated at different moments, the two droplets in some cases will go into a precession state with completely opposite phase. In this case the merger process will not occur. The details of this opposite behavior are, however, beyond the scope of this work.

In summary, we have investigated the dynamics of a droplet pair merging into a single droplet under specific combinations of applied current and magnetic field. The frequency of the merged droplet is a little lower than that of the droplet pair, in agreement with theory. The merging only occurs for NC separations below a current and field dependent critical value. The inverse process from a larger merged droplet back to a droplet pair can be realized by increasing the external magnetic field. These results provide insight into a fundamental micromagnetic process that could be useful for spin torque oscillators and magnonic devices based on magnetic droplet solitons.

This work is supported by the National Basic Research Program of China (Grant No. 2015CB921501) and by the National Natural Science Foundation of China (Grants No. 51471118 and No. 11274241). Y.Z. acknowledges support by the National Natural Science Foundation of China (Project No. 1157040329), the Seed Funding Program for Basic Research and Seed Funding Program for Applied Research from the HKU, ITF Tier 3 funding (ITS/171/13, ITS/203/14), and from the RGC-GRF under Grant No. HKU 17210014. 
[1] A. M. Kosevich, B. A. Ivanov, and A. S. Kovalev, Phys. Rep. 194, 117 (1990).

[2] H.-B. Braun, Adv. Phys. 61, 1 (2012).

[3] V. S. Pribiag, I. N. Krivorotov, G. D. Fuchs, P. M. Braganca, O. Ozatay, J. C. Sankey, D. C. Ralph, and R. A. Buhrman, Nat. Phys. 3, 498 (2007).

[4] A. Dussaux et al., Nat. Commun. 1, 8 (2010).

[5] K. Yamada, S. Kasai, Y. Nakatani, K. Kobayashi, H. Kohno, A. Thiaville, and T. Ono, Nat. Mater. 6, 270 (2007).

[6] A. Abanov and V. L. Pokrovsky, Phys. Rev. B 58, R8889 (1998).

[7] B. A. Ivanov, V. A. Stephanovich, and A. A. Zhmudskii, J. Magn. Magn. Mater. 88, 116 (1990).

[8] A. Fert, V. Cros, and J. Sampaio, Nat. Nanotechnol. 8, 152 (2013).

[9] N. Nagaosa and Y. Tokura, Nat. Nanotechnol. 8, 899 (2013).

[10] N. L. Schryer and L. R. Walker, J. Appl. Phys. 45, 5406 (1974).

[11] T. C. Chen and H. Chang, Adv. Comput. 17, 223 (1979).

[12] P. Dekker and J. C. Slonczewski, Appl. Phys. Lett. 29, 753 (1976).

[13] A. P. Malozemoff and J. C. Slonczewski, in Magnetic Domain Walls in Bubble Materials, edited byA. P. M. C. Slonczewski (Academic, New York, 1979), p. 217.

[14] A. Thiaville, J. M. Garciía, and J. Miltat, J. Magn. Magn. Mater. 242, Part 2, 1061 (2002).

[15] S. S. P. Parkin, M. Hayashi, and L. Thomas, Science 320, 190 (2008).

[16] T. Shinjo, T. Okuno, R. Hassdorf, K. Shigeto, and T. Ono, Science 289, 930 (2000).

[17] K. Y. Guslienko and V. Novosad, J. Appl. Phys. 96, 4451 (2004).

[18] K. Y. Guslienko and K. L. Metlov, Phys. Rev. B 63, 100403 (2001).

[19] Y. Liu, S. Gliga, R. Hertel, and C. M. Schneider, Appl. Phys. Lett. 91, 112501 (2007).

[20] Y. Liu, Z. Hou, S. Gliga, and R. Hertel, Phys. Rev. B 79, 104435 (2009).
[21] Y. Zhou, E. Iacocca, A. A. Awad, R. K. Dumas, F. C. Zhang, H B. Braun, and J. Åkerman, Nat. Commun. 6, 8193 (2015).

[22] M. A. Hoefer, T. J. Silva, and M. W. Keller, Phys. Rev. B 82, 054432 (2010).

[23] M. A. Hoefer, M. Sommacal, and T. J. Silva, Phys. Rev. B 85, 214433 (2012).

[24] S. M. Mohseni et al., Science 339, 1295 (2013).

[25] S. Chung et al., J. Appl. Phys. 115, 172612 (2014).

[26] F. Macià, D. Backes, and A. D. Kent, Nat. Nanotechnol. 9, 992 (2014).

[27] S. Chung, A. Eklund, E. Iacocca, S. M. Mohseni, S. R. Sani, L. Bookman, M. A. Hoefer, J. Åkerman, and R. K. Dumas (unpublished).

[28] A. Slavin and V. Tiberkevich, Phys. Rev. Lett. 95, 237201 (2005).

[29] T. J. Silva and W. H. Rippard, J. Magn. Magn. Mater. 320, 1260 (2008).

[30] R. K. Dumas et al., IEEE Trans. Magn. 50, 1 (2014).

[31] J. C. Slonczewski, J. Magn. Magn. Mater. 159, L1 (1996).

[32] L. Berger, Phys. Rev. B 54, 9353 (1996).

[33] S. M. Mohseni et al., Physica B 435, 84 (2014).

[34] S. Lendínez, N. Statuto, D. Backes, A. D. Kent, and F. Maciá, Phys. Rev. B 92, 174426 (2015).

[35] A. Vansteenkiste, J. Leliaert, M. Dvornik, M. Helsen, F. GarciaSanchez, and B. Van Waeyenberge, AIP Adv. 4, 107133 (2014).

[36] X. Li, Z. Zhang, Q. Y. Jin, and Y. Liu, New J. Phys. 11, 023027 (2009).

[37] E. Iacocca, R. K. Dumas, L. Bookman, M. Mohseni, S. Chung, M. A. Hoefer, and J. Åkerman, Phys. Rev. Lett. 112, 047201 (2014).

[38] See Supplemental Material at http://link.aps.org/supplemental/ 10.1103/PhysRevB.93.094431 for perspective view of the magnetization with a dynamic structure from a droplet pair to a larger merged droplet.

[39] A. M. Kosevich, B. A. Ivanov, and A. S. Kovalev, Physica D 3, 363 (1981). 